\title{
Retrieval of Associative Information Congruent with Prior Knowledge Is Related to Increased Medial Prefrontal Activity and Connectivity
}

\author{
Marlieke T. R. van Kesteren, ${ }^{1,2}$ Mark Rijpkema, ${ }^{1}$ Dirk J. Ruiter, ${ }^{2}$ and Guillén Fernández ${ }^{1,3}$ \\ ${ }^{1}$ Radboud University Nijmegen, Donders Institute for Brain, Cognition, and Behaviour, and Radboud University Nijmegen Medical Centre, Departments of \\ ${ }^{2}$ Anatomy and ${ }^{3}$ Cognitive Neuroscience, $6500 \mathrm{HB}$, Nijmegen, the Netherlands
}

We remember information that is congruent instead of incongruent with prior knowledge better, but the underlying neural mechanisms related to this enhancement are still relatively unknown. Recently, this memory enhancement due to a prior schema has been suggested to be based on rapid neocortical assimilation of new information, related to optimized encoding and consolidation processes. The medial prefrontal cortex (mPFC) is thought to be important in mediating this process, but its role in retrieval of schema-consistent information is still unclear. In this study, we regarded multisensory congruency with prior knowledge as a schema and used this factor to probe retrieval of consolidated memories either consistent or inconsistent with prior knowledge. We conducted a visuotactile learning paradigm in which participants studied visual motifs randomly associated with word-fabric combinations that were either congruent or incongruent with common knowledge. The next day, participants were scanned using functional magnetic resonance imaging while their memory was tested. Congruent associations were remembered better than incongruent ones. This behavioral finding was parallelized by stronger retrieval-related activity in and connectivity between medial prefrontal and left somatosensory cortex. Moreover, we found a positive across-subject correlation between the connectivity enhancement and the behavioral congruency effect. These results show that successful retrieval of congruent compared to incongruent visuotactile associations is related to enhanced processing in an mPFCsomatosensory network, and support the hypothesis that new information that fits a preexisting schema is more rapidly assimilated in neocortical networks, a process that may be mediated, at least in part, by the mPFC.

\section{Introduction}

New information that is consistent with prior knowledge is remembered better. Why this enhancement arises, and how it is expressed in the brain, however, is still relatively unknown. Prior knowledge is suggested to lead to easier assimilation within an interrelated set of neocortical representations, or schema, when this new information finds multiple links within such a schema (Wang and Morris, 2010). Neural processes related to optimized encoding (van Kesteren et al., 2010) and consolidation (Tse et al., 2007; Wang and Morris, 2010) have been suggested to play an important role in this enhancement. However, how prior knowledge affects processes related to memory retrieval has not yet been investigated.

Along with the hippocampus, an important brain region that is indicated to be involved in the retrieval of consolidated memories is the medial prefrontal cortex (mPFC) (Frankland and Bontempi, 2006; Takashima et al., 2006; Takehara-Nishiuchi and McNaughton, 2008). Moreover, the interaction between the hip-

Received May 25, 2010; revised Sept. 13, 2010; accepted Sept. 28, 2010.

We thank Vincent $S$ choots for graphical assistance.

Correspondence should be addressed to Marlieke T. R. van Kesteren, Donders Institute for Brain, Cognition, and Behaviour, Centre for Cognitive Neuroimaging, P.0. Box 9101, 6500 HB Nijmegen, The Netherlands. E-mail: marlieke.vankesteren@donders.ru.nl.

DOI:10.1523/JNEUROSCI.2674-10.2010

Copyright $\odot 2010$ the authors $\quad 0270-6474 / 10 / 3015888-07 \$ 15.00 / 0$ pocampus and the mPFC is believed to be important already in early stages of memory formation, as discovered in rodents (Benchenane et al., 2010), as well as humans (van Kesteren et al., 2010). Specifically, hippocampal-mPFC coupling is found to decrease during encoding of new information that fits prior knowledge (van Kesteren et al., 2010), suggesting that along with hippocampal involvement, the mPFC may play an important role already during initial processing of schema-related information. Based on these insights, the pointer function of the hippocampus, binding distributed memory representations (Marr, 1970; Rasch and Born, 2007), has been suggested to gradually shift to the mPFC (Frankland and Bontempi, 2006; Takashima et al., 2006), a process that is potentially facilitated by a preexisting schema (van Kesteren et al., 2010). Consequently, retrieval of a stimulus consistent with prior schema is expected to lead to improved memory performance (Bransford and Johnson, 1972; Staresina et al., 2009), related to enhanced mPFC activity and connectivity with specific brain areas, representing elements of the learned information (Wheeler et al., 2000).

In this study, we investigated the effect of preexisting schema on retrieval-related brain activity by manipulating semantic congruency of multisensory stimuli (Kim et al., 2008; Shams and Seitz, 2008). We chose for this setup, because multisensory stimuli that fit with prior knowledge can be regarded as schemacongruent, and remembering multisensory information is easier if it represents a feature combination congruent with prior expe- 
Day 1

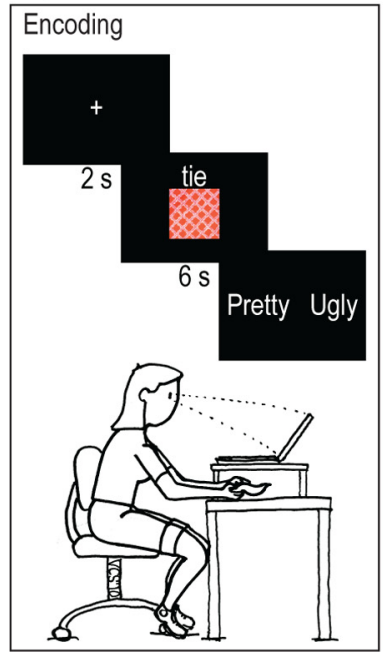

Day 2

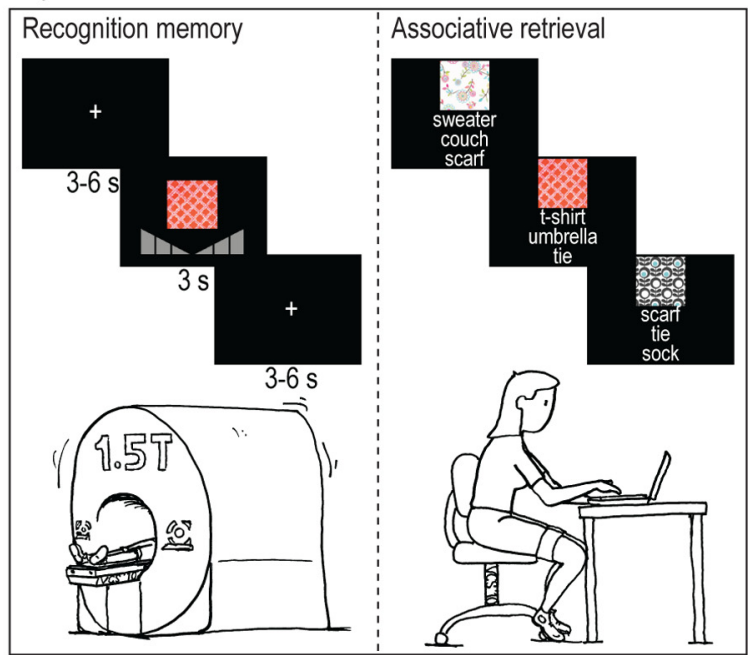

Figure 1. Experimental design. On day 1, participants learned associations of visual motifs and congruent or incongruent object-fabric combinations, where the object was presented together with the motif as a written word on the computer screen, and the fabric simultaneously as a tactile stimulus underneath the computer screen. On day 2, participants were tested in the MR scanner by means of a visual item recognition test (motifs) and subsequently with an associative memory test outside the MR scanner in which the motifs served as cues and the associated word was asked for in a three-choice test.

rience (Kim et al., 2008; Yuval-Greenberg and Deouell, 2009). This semantic congruency can be regarded as information that can readily be assimilated into preexisting mental schemata. If this hypothesis holds, semantic congruency of multisensory stimuli will act as a schema and lead to enhanced memory performance along with enhanced activity in and connectivity between the mPFC and specific sensory cortices at retrieval (Harris et al., 2001). Concomitant with this mPFC enhancement, hippocampal involvement at retrieval is expected to decrease.

\section{Materials and Methods}

Participants. Twenty-six native Dutch female right-handed students participated in this study. All were healthy and had normal or corrected-tonormal vision. They were paid to participate and were told that they could earn extra money for better performance. Three participants were excluded after data acquisition, one because of excessive movement during scanning, and two because of poor item memory performance (total item recognition hits $<30$ ), which left 23 participants for analyses. This sample covered an age range of $18-30$ years, with a mean age of 22.65 years. They self-reported to have slept on average $7.67 \mathrm{~h}$ in between the 2 examination days (ranging from 6 to $9 \mathrm{~h}$ ). We decided to recruit women only, because they generally have more interest in and knowledge about fashion-like stimuli, and they are shown to have more tactile spatial acuity in their fingertips than men (Peters et al., 2009). Ethical approval was obtained from the institutional review board (CMO Region Arnhem-Nijmegen, The Netherlands), and all participants gave written informed consent.

Stimuli. Participants learned a series of triplets of simultaneously presented stimuli that, when associated with each other, formed an object likely to be present in real life. These associations consisted of (1) motifs (200), visually presented as a two-dimensional, pictorial square without tactile information; (2) visually presented object words (20) describing objects primarily composed of fabrics; and (3) fabric samples (20) that could be linked to the object words. Motifs (400 in total, including lures) were obtained from the Internet, and were equalized in size $(256 \times 256$ pixels, 28.35 pixels $/ \mathrm{cm}$, indexed color mode) and autocontrasted using Adobe Photoshop CS3, version 10.0.1 (Adobe). Fabric samples were cut into squares of $5 \times 5 \mathrm{~cm}$, and object-fabric combinations were categorized as being either semantically congruent (for example, a leather jacket) or semantically incongruent (for example, a lace umbrella). The (in)congruency of these combinations was verified in an independent behavioral pilot, where participants $(n=12)$ were asked to rate the congruency of wordfabric combinations from 1 to 6 . Combinations rated on average 2.5 or lower were considered incongruent, and combinations rated on average 3.5 or higher were considered congruent. Combinations in between these ratings were altered to either fit a congruent or incongruent representation.

Design and general procedure. Participants were tested using a within-subject $2 \times 3$ factorial design with congruency (congruent items vs incongruent items) and memory (associatively remembered items vs associatively forgotten items vs completely forgotten items) as within-subject factors (see Fig. 1). They were invited to come to the center on 2 consecutive days with on average $19.83 \mathrm{~h}$ between the two visits (ranging from 18.5 to $21 \mathrm{~h}$ ). On day 1 , participants were instructed to memorize simultaneously presented triplets of visual motifs, visual object words, and tactile fabric samples by imagining how the combination of these features would look. They were told that their memory would be tested in the MR scanner on the next day, but they received no information about the specifics of this memory test. Using Presentation 10.2 (Neurobehavioral Systems), the motif and the word were visually presented on a computer screen for $6 \mathrm{~s}$, the word situated above the motif. Concurrently, participants were instructed in a practice session to tactilely explore a fabric for the complete $6 \mathrm{~s}$, and imagine how the combination of motif, word, and fabric would look. The fabric was presented by the experimenter underneath a heightened plateau on which the computer screen was placed, and was not visible to the participant. After presentation of each stimulus combination, participants were asked to indicate whether they thought the triplet characterizing the imagined object was either pretty or ugly (see Fig. 1).

In total, participants memorized 200 sequentially presented combinations, 100 congruent and 100 incongruent, divided into three sessions of consecutively 80,80 , and 40 trials. The 20 object words and 20 fabric samples were combined into 80 possible combinations, with each object word linked to two congruent and two incongruent fabrics. For the last session of 40 presentations, only one congruent and one incongruent object-fabric combination were used instead of two. Within each session, object-fabric associations were randomly divided, but equal for each participant, while motifs were randomly shuffled for each participant. Thus, every participant learned the same object-fabric combinations, but for each participant these were differently associated with the motifs.

On the second day, participants were scanned while they performed an item recognition memory test (with confidence rating) for the motifs presented the day before. Participants lay supine in the scanner, wore headphones (Commander XG, Magnetic Resonance Technology), and responded using left and right button boxes, which were fastened with tape to the upper legs so they would not move. They viewed the screen through a mirror positioned on top of the head coil. They were instructed to respond within the $3 \mathrm{~s}$ presentation time, try to move as little as possible, and keep their fingers as still as possible to avoid involvement of somatosensory stimulation during button presses. Participants received a practice session before starting the experiment. Stimuli were presented in the center of the screen for $3 \mathrm{~s}$, and were followed by a fixation cross, presented for 3-6 s. Furthermore, 10 fixation cross baseline trials of $10 \mathrm{~s}$ duration were included. These baseline trials were distributed so that within every 40 trials, a baseline trial was presented. After the item recognition memory test, which lasted $51 \mathrm{~min}$ and $20 \mathrm{~s}$, a structural scan of $9 \mathrm{~min}$ and $38 \mathrm{~s}$ was made. Finally, a localizer for the somatosensory cortex (six cycles of a simple blocked design; $15 \mathrm{~s}$ on, $15 \mathrm{~s}$ off) was performed where participants received somatosensory stimulation on all fingers of 
both hands by simultaneously moving two cotton swabs across their fingers. This localizer scan lasted $3 \mathrm{~min}$ and $15 \mathrm{~s}$ in total, and was performed to identify a region of interest (ROI) for further analyses, since the somatosensory cortex has been suggested to be the primary location of somatosensory memories (Burton and Sinclair, 2000; Gallace and Spence, 2009). After the scan session, participants were taken to another room to perform an associative retrieval task additionally.

Memory tests and analyses. Item recognition memory was tested in the MR scanner using a confidence level approach (six levels) in which participants were instructed to indicate whether a perceived stimulus (200 old and 200 new) was old or new. Six answer options were provided: sure old, nearly sure old, not sure old, not sure new, nearly sure new, and sure new. Answers were given with left and right ring fingers, middle fingers, and index fingers, with the old/new side counterbalanced across participants. The order of the motifs was pseudorandom; no more than four consecutive old or new stimuli were presented. Participants could only answer once and were given feedback on which button they pressed. Answers that were given too late (i.e., after the 3 s presentation time) or were indicated as not sure were not included in the analyses.

Subsequent to the item recognition memory test, participants performed a self-paced, three-alternative forced-choice associative memory task outside the scanner, in which they were instructed to indicate which object word was associated with a certain motif on the previous day. All 200 memorized motifs were randomly and sequentially presented on a computer screen as cues, together with three words, of which one word was the correct answer, and the two other words were randomly sampled from the other 19 words. After participants finished this test, they filled out a study-specific questionnaire.

Behavioral measures of item recognition scores were analyzed using SPSS 15.0 (SPSS) by calculating the percentage of hits and false alarms (both sure old and nearly sure old confidence levels) for both conditions (congruent and incongruent). Next, these values were $z$-transformed and subtracted from each other to calculate $d^{\prime}$ for both conditions. Subsequently, Student's $t$ tests were performed to determine differences from chance level ( 0 ; one-sample $t$ test) and differences between the congruent and incongruent conditions (paired-sample $t$ test). Associative memory was analyzed using only the items that were correctly recognized during item recognition. Of these items, percentage correct was calculated for both conditions, and again one-sample (with chance level 1/3) and paired-sample Student's $t$ tests were performed to determine congruency differences. Also, in both memory tests, reaction time differences between both conditions were assessed using the same statistical tests. $\alpha$ was set at 0.05 throughout.

MRI scanning parameters. Participants were scanned using a 1.5 tesla Siemens Magnetom Avanto system equipped with an eight-channel phased array head coil (MRI Devices). For blood oxygen level-dependent (BOLD) functional magnetic resonance imaging (fMRI) images, we used a T2*-weighted gradient echo planar imaging sequence with the following parameters: repetition time (TR): $2.48 \mathrm{~s}$, echo time: $35 \mathrm{~ms}$, 34 slices, ascending slice order, $3.5 \mathrm{~mm}$ slice thickness, $0.35 \mathrm{~mm}$ slice gap, matrix size: $64 \times 64$, field of view (FOV): $212 \times 212 \mathrm{~mm}$, flip angle: $90^{\circ}$, and voxel size: $3.3 \times 3.3 \times 3.5$. Slices were angulated in an oblique axial manner to reach whole-brain coverage. To ensure reaching a steady-state condition, the first five scans were discarded. Additionally, T1-weighted anatomical scans at $1 \mathrm{~mm}$ isotropic resolution were acquired with TR of $2250 \mathrm{~ms}$, inversion time of $850 \mathrm{~ms}$, flip angle of $15^{\circ}$, and FOV of $256 \times$ $256 \times 176 \mathrm{~mm}$.

fMRI data preprocessing and analyses. Raw fMRI data were preprocessed using SPM5 (http://www.fil.ion.ucl.ac.uk/spm). First, motion correction was performed by using iterative rigid body realignment to minimize the residual sum of squares between the first and all further functional scans. Then, ascending slice timing correction was performed such that all slices were corrected to the time of acquisition of the reference slice (i.e., the middle slice, 17). Subsequently, rigid body coregistration to corresponding individual T1 images was performed using mutual information optimization. Hereafter, data were spatially normalized into a common space, defined by the Montreal Neurological Institute $152 \mathrm{~T} 1$ image (voxel size $=2 \times 2 \times 2$ ), and smoothed by convolving the data with an $8 \mathrm{~mm}$ full-width at half-maximum three-dimensional kernel. The first five scans were excluded, which left 1242 scans for analysis.

After preprocessing, statistical parametric maps were generated by modeling the evoked BOLD response for each memory bin (associative hits: item hit + association remembered; associative misses: item hit + association forgotten; forgotten: item forgotten) as a boxcar function of 3 s convolved with a hemodynamic response function. Furthermore, individual movement regressors were added to each first-level model. Subsequently, a random-effects $2 \times 3$ factorial design was constructed in which congruency (congruent and incongruent) could be tested against memory (item hit + association remembered; item hit + association forgotten; item forgotten). Whole-brain activity for main and interaction effects was considered significant at $p<0.05$ corrected at cluster level, after creating a $p<0.001$ uncorrected map or small volume corrected (SVC) at $p<0.05$ based on a $p<0.001$ uncorrected map, with independently determined regions of interests: bilateral hippocampi taken from the AAL template (Tzourio-Mazoyer et al., 2002) and the somatosensory localizer (left side).

The localizer scans were processed using the same procedures as the experimental scans, but without slice time correction. Hemodynamic responses were modeled using a statistical parametric map in which blocks of $15 \mathrm{~s}$ on/off were modeled as boxcar functions convolved with a hemodynamic response function, and individual movement regressors were again added to each first-level model. To assess a random-effects analysis of somatosensory stimulation, a one-sample $t$ test was performed. Because activity appeared very strong, we used a threshold of $p<$ 0.00005 uncorrected for this analysis. To determine whether activation was close to the region in the somatosensory cortex dedicated to the fingers, we furthermore used coordinates from a previous study revealing these regions for the separate fingers (Weibull et al., 2008), surrounded by a $6 \mathrm{~mm}$ sphere, as ROI ( $p<0.001$ uncorrected; $p<0.05$ SVC).

Psychophysiological interactions (PPIs) were calculated to assess functional connectivity between regions. They were executed using SPM5 (http://www.fil.ion.ucl.ac.uk/spm) in combination with in-house software, written in Matlab 7.5 (The MathWorks). Psychophysiological interaction analyses probe differences in coactivation of a certain seed region (physiological factor) with the rest of the brain modulated by an external factor (psychological factor). Here, we examined coactivation differences that were significantly larger for congruent than for incongruent items and vice versa. Only voxels that were significantly active in an effect of interest analysis were used for this analysis. The single-subject general linear model (GLM) constructed for previous analyses was extended with two regressors: the general deconvolved signal from the seed region, and the deconvolved signal from the seed region for the contrast congruent hits versus incongruent hits. For each subject, this second physiological activity was used as input for the second-level randomeffects analysis. The seeds in mPFC and somatosensory cortex, present in the congruency $\times$ subsequent associative memory interaction, were defined by taking the peak voxel surrounded by a $5 \mathrm{~mm}$ sphere. Connectivity was again considered significant at $p<0.001$ uncorrected at voxel level and $p<0.05$ corrected at cluster level or at $p<0.001$ uncorrected at voxel level and SVC at $p<0.05$ with the somatosensory localizer.

To determine correlations between PPI strength and behavior across participants, PPI measures were extracted from SPM and analyzed using SPSS. A two-tailed Pearson correlation test between PPI measures and congruency benefit of associatively remembered items (congruent/incongruent), was performed. $\alpha$ was again set at 0.05 .

\section{Results}

\section{Memory performance}

Memory performance measures showed a semantic congruency effect on associative memory $\left(t_{(22)}=4.09, p<0.001\right)$, with both measures different from chance level [congruent items: $t_{(22)}=$ 8.16, $p<0.001$ (mean $=0.51 ; \mathrm{SD}=0.11$ ), incongruent items: $t_{(22)}=5.30, p<0.001$ (mean $\left.\left.=0.43 ; \mathrm{SD}=0.09\right)\right]$. Also, item recognition memory for the visually presented motifs showed a congruency effect $\left(t_{(22)}=2.13, p<0.05\right)$, and were different from chance $\left[\right.$ congruent items: $t_{(22)}=9.97, p<0.001$ ( mean $=$ 


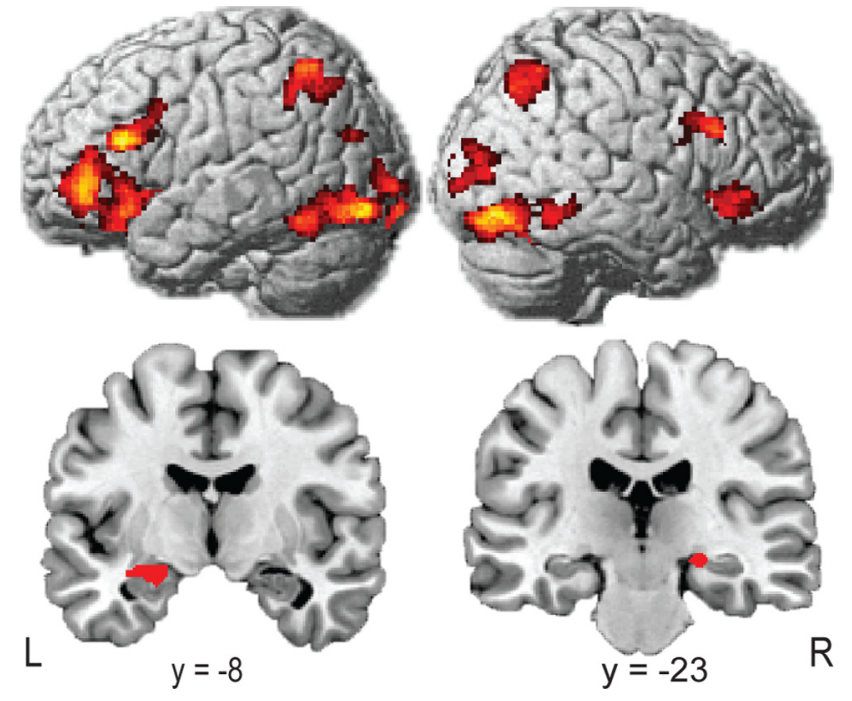

Figure 2. Main effect of associative memory. GLM analyses revealed a set of brain regions comprised of dorsal and ventral visual areas, inferior parietal sulci, dorsolateral and ventrolateral prefrontal cortices (cluster-level corrected; $p<0.05$ ), and bilateral hippocampi ( $p<0.05$ SVC corrected; peaks $[-32,-10,-12]$ and $[22,-22,-10])$.

$0.80 ; \mathrm{SD}=0.38)$, incongruent items: $t_{(22)}=9.32 ; p<0.001$ $($ mean $=0.71 ; \mathrm{SD}=0.36)]$. Reaction times during both item recognition and associative memory were not different in either of these bins $\left(t_{(22)}=0.52, p>0.05\right.$, n.s.; $t_{(22)}=1.67, p>$ 0.05 , n.s.).

\section{Neuroimaging results: differential activity}

When analyzing fMRI data related to successful recognition memory differing in terms of correct associative retrieval (associative hits vs associative misses) we revealed a set of brain regions encompassing bilateral hippocampus (peaks $[-32,-10,-12]$ and $[22,-22,-10]$; SVC corrected) (Fig. 2), areas in the dorsal and ventral visual streams, inferior parietal sulci, dorsolateral and ventrolateral prefrontal cortices, and basal ganglia (cluster-level corrected) (Fig. 2). When performing a congruency $\times$ associative memory ANOVA, testing activity differences for congruent $>$ incongruent and associatively remembered $>$ associatively forgotten, an interaction in the $\mathrm{mPFC}$, extending from anterior cingulate cortex [Brodmann's area (BA) 32] into BA 10 was found. This interaction was based on larger differential responses for congruent as opposed to incongruent trials (peak $=[-6,34,12]$; cluster-level corrected) (Fig. 3). Another cluster of interacting voxels was found within the left hemispheric region activated by the somatosensory localizer scan (peak $[-50,-20,32]$; clusterlevel corrected) (Fig. 3). This cluster was located within a sphere with $6 \mathrm{~mm}$ radius surrounding an area in the somatosensory cortex previously found to be related to sensory sensation in the thumb [-52, -19, 42] (Weibull et al., 2008). No significant effects were observed for the opposite contrast.

\section{Neuroimaging results: differential connectivity}

To assess connectivity differences, we performed PPI analyses on the $\mathrm{mPFC}$ cluster found in the congruency $\times$ subsequent associative memory interaction for congruent versus incongruent associative hits. This seed region revealed significant coactivation with the left somatosensory cortex (peak [-56, - 16, 48]; SVC corrected) (Fig. 3), which was stronger for congruent associative hits than incongruent associative hits. Again, this cluster was within a sphere with $6 \mathrm{~mm}$ radius surrounding the areas in the somatosensory cortex related to sensory sensation in the thumb (Weibull et al., 2008). No significant effects were observed for the opposite contrast.

\section{Brain-behavior relation}

We next tested whether across participants this congruency effect on mPFC connectivity predicted the behavioral benefit in terms of memory performance. Here, we found that the PPI between the $\mathrm{mPFC}$ and the left somatosensory cortex for the congruent versus the incongruent associatively remembered items predicted the congruency benefit of associatively remembered items (congruent/incongruent) $\left(r_{(22)}=0.531 ; p<0.01\right)$ (Fig. 3).

\section{Discussion}

The current results show that mPFC activity and connectivity with a specific representational cortical area is enhanced when multisensory learned information is retrieved that is congruent with a general, preexisting mental schema. Additionally, this increase in functional connectivity was found to be positively correlated to the behavioral benefit associated with this preexisting knowledge across participants. These results are in line with a model in which the $\mathrm{mPFC}$ is involved in readily assimilating new information into preexisting schemata during memory formation and consolidation (van Kesteren et al., 2010; Wang and Morris, 2010).

Behaviorally, our results show enhanced memory for semantically congruent compared to incongruent multisensory associations. This memory-enhancing effect of semantic congruency has previously been identified (Yuval-Greenberg and Deouell, 2009), but its underlying neural mechanisms were largely unknown. This effect has previously been suggested to be related to working memory mechanisms (Zwaan and Radvansky, 1998), or intertwined encoding and consolidation processes during and immediately after learning (van Kesteren et al., 2010). The present data show congruency effects in the mPFC during retrieval processes. Therefore, along with working memory, encoding, and postlearning consolidation, distinct retrieval processes may be related to this behavioral advantage. Our results can thus be explained by adopting a framework where modulation of both learning and postlearning processes leads to long-term modulation of memory traces, with semantic congruency allowing new information to be faster and better embedded into an existing associative mnemonic network (Tse et al., 2007; van Kesteren et al., 2010; Wang and Morris, 2010).

The congruency effect that we found at the behavioral level was expressed in within-subject activity and connectivity differences in $\mathrm{mPFC}$ and left somatosensory cortex, and was additionally related to connectivity differences between these regions across participants. These results confirm that the MPFC plays a key role in retrieving consolidated memories (Frankland and Bontempi, 2006; Takashima et al., 2006; Takehara-Nishiuchi and McNaughton, 2008). Additionally, since semantic congruency reflects the degree to which the newly learned information fits to preexisting knowledge (i.e., schema) (Kim et al., 2008), and given the assumption that such congruent information is more rapidly assimilated than information that does not fit a prior schema (Tse et al., 2007; van Kesteren et al., 2010), our data further support the view that such a fast track in memory consolidation might also be present in humans.

Additionally, our results partly support the hypothesis that the pointer function of the hippocampus, binding distributed memory representations (Marr, 1970; Rasch and Born, 2007), shifts to the mPFC (Frankland and Bontempi, 2006; Takashima 


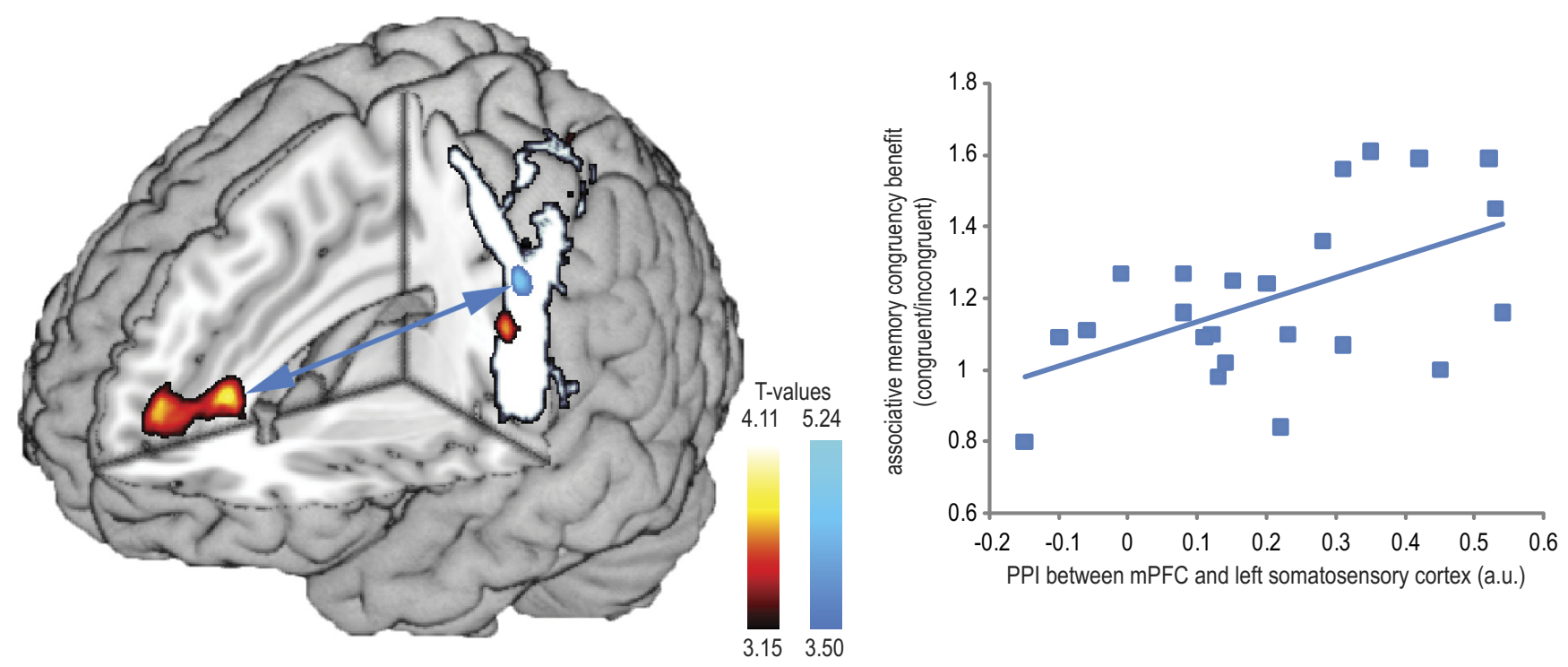

Figure 3. Congruency $\times$ associative subsequent memory interaction and PPI results from mPFC. The congruency $\times$ associative subsequent memory interaction showed activity (red/yellow) in $\mathrm{mPFC}(p<0.01$ cluster-level corrected), and in the left somatosensory cortex ( $p<0.05$ cluster-level corrected) (overlaid on the localizer scan in white/gray). A psychophysiological analysis with $\mathrm{mPFC}$ as seed region showed a significant coactivation (blue arrow) with the left somatosensory cortex, which was stronger for congruent hits than for incongruent hits (blue). Finally, a correlation between mPFC-somatosensory coupling and behavioral congruency benefit was found. The more connectivity present during item recognition of associatively remembered items, the higher the congruency benefit for subsequent associative retrieval $\left(r_{(22)}=0.531 ; p<0.01\right)$.

et al., 2006). This functional balance is suggested to be facilitated by prior knowledge (van Kesteren et al., 2010). However, this hypothesis predicts a reduction in hippocampal contribution to memory retrieval of congruent stimuli along with an increase in $\mathrm{mPFC}$ contribution, which we did not find. One explanation for this null finding related to hippocampal processing and connectivity may be that we scanned memory retrieval processes after only one day/night cycle (on average $20 \mathrm{~h}$ later). Although systems consolidation mechanisms have been indicated to be more rapid than previously assumed (Takashima et al., 2006; Diekelmann and Born, 2010), and facilitated by prior knowledge (Tse et al., 2007; van Kesteren et al., 2010), these mechanisms are still very likely to abide in an early phase as observed here. Therefore, retrieving the memory trace still requires hippocampal mediation for both congruent and incongruent associations. Further research will be needed to reveal whether hippocampal activity will decrease in time (Takashima et al., 2006, 2009), or whether the hippocampus remains activated while such associative memories are retrieved (Moscovitch et al., 2006), regardless of the occurrence of a (partial) shift of its pointer function to the mPFC.

Similar to the hippocampus, the function of the mPFC in consolidation is broadly investigated while many questions remain. Along with its putative role in systems consolidation and retrieval (Maguire, 2001; Frankland and Bontempi, 2006; Takashima et al., 2006; Takehara-Nishiuchi and McNaughton, 2008), the mPFC has been indicated as a region involved in a diversity of functions, among which many with a mnemonic nature. The prefrontal cortex in general is believed to be involved in updating, maintenance, and manipulation of memory traces (Buckner and Wheeler, 2001; Fletcher and Henson, 2001), and the mPFC in particular is thought to be related to feeling of knowing (Kikyo et al., 2002), conceptual knowledge integration (Kumaran et al., 2009), perceptual matching (Summerfield and Koechlin, 2008), comprehension (Maguire et al., 1999; Mar, 2004), and remote associative memory (Takashima et al., 2007; Takehara-Nishiuchi and McNaughton, 2008), and is shown to actively replay learning-related neuronal spiking patterns during sleep (Takehara-Nishiuchi and McNaughton, 2008; Peyrache et al., 2009). Furthermore, mPFC lesions lead to specific retrieval impairments for remote, presumably consolidated memories (Takehara-Nishiuchi et al., 2006; Gilboa et al., 2009), and an absence of semantic congruency memory enhancement (Kan et al., 2009). Finally, its connectivity to several other brain regions makes it very well suited to retrieve distributed memory traces (Miyashita, 2004). This accumulating line of evidence clearly shows the critical importance of the (m)PFC in all stages of mnemonic brain functioning.

In contrast with its mnemonic function, more dorsal parts of the mPFC have also been suggested to be involved in rather general control mechanisms such as error monitoring and effort related to task difficulty (Ridderinkhof et al., 2004). However, since enhanced memory performance in our study is positively related to larger mPFC activity, it is unlikely that the mPFC activity observed during remote memory retrieval in this and previous studies (Takashima et al., 2006, 2007) is related to such general control function of the mPFC. In sum, our findings extend current literature on the mnemonic function of the $\mathrm{mPFC}$ by showing its congruency-dependent involvement in remote memory retrieval, and support the view that the mPFC offers a fast track into consolidated memories if newly stored information fits preexisting schemata (Frankland and Bontempi, 2006; van Kesteren et al., 2010).

The presence of prior knowledge is widely known to enhance memory of new information that fits this knowledge (Bransford and Johnson, 1972), but hitherto one could only speculate on the actualization of this memory enhancement. Our data elucidate some of the underlying neural mechanisms of this process. How a prior associative schema enhances memory formation, however, remains to be determined. A plausible hypothesis is that two different learning systems (focused/fast learning vs interleaved/slow learning) are present in the brain, as proposed by McClelland et al. (1995). We suggest, based on this theory and our data, that when new information 
is inconsistent with prior knowledge, more interleaved learning is necessary to assimilate this information in preexisting memory networks. In turn, consistent information needs less interleaved learning to be adequately integrated, speeding up assimilation and concurrent $\mathrm{mPFC}$ involvement. In this view, the presence of prior knowledge can modulate the processing speed of consistent incoming information due to less necessity to interleave it with inconsistent prior memory networks.

Learning of multisensory perceived stimuli, as applied here, is a relatively underinvestigated area of learning and memory (Shams and Seitz, 2008). Here, by modulating congruency, multisensory learning proved a very efficient tool to investigate mnemonic mechanisms at retrieval, but might accordingly also be used to investigate encoding and consolidation mechanisms. In particular, since associative sensory features of a learned stimulus are found to reactivate sensory areas at retrieval (Harris et al., 2001), even when only one sensory modality is cued (Nyberg et al., 2000; von Kriegstein and Giraud, 2006), these reactivations can reliably probe binding of modality-specific distributed brain regions while retrieving relevant information, either hippocampally (Staresina et al., 2009; Takashima et al., 2009) or neocortically mediated (as reported here). Furthermore, the assimilation of multisensory perceived stimuli into one coherent whole (Amedi et al., 2005; Driver and Noesselt, 2008), can be more thoroughly investigated when considering long-term consequences of these assimilative mechanisms (Shams and Seitz, 2008), and the mediating effect of (semantic) congruency (Kim et al., 2008; Yuval-Greenberg and Deouell, 2009). Finally, since training can modify congruency judgments (Ernst, 2007), sometimes even modulated by other modalities (Fredembach et al., 2009), these findings can be very helpful when designing educational programs where multisensory learning is an integral part of the curriculum (Lasry and Aulls, 2007) [e.g., in medical education (Patel et al., 2009)].

These results provide support for the view that the MPFC is crucially involved in retrieval of consolidated associative memories, and the role of prior knowledge in these mechanisms. By modulating prior knowledge, this paradigm shows that we can gain more insight into how new information is assimilated in preexisting knowledge networks. This insight is of crucial importance to advance mnemonic research to memory formation, consolidation, and retrieval. Furthermore, the memory-enhancing effect related to the facilitatory nature of prior knowledge is of great importance to educational strategies. If educators understand more about the mechanisms underlying this facilitation, educational programs can be better structured, leading to more efficient learning in classroom settings (Bransford et al., 2000).

\section{References}

Amedi A, von Kriegstein K, van Atteveldt NM, Beauchamp MS, Naumer MJ (2005) Functional imaging of human crossmodal identification and object recognition. Exp Brain Res 166:559-571.

Benchenane K, Peyrache A, Khamassi M, Tierney PL, Gioanni Y, Battaglia FP, Wiener SI (2010) Coherent theta oscillations and reorganization of spike timing in the hippocampal-prefrontal network upon learning. Neuron 66:921-936.

Bransford JD, Johnson MK (1972) Contextual prerequisites for understanding - some investigations of comprehension and recall. J Verb Learn Verb Behav 11:717-726.

Bransford JD, Brown AL, Cocking RR (2000) How people learn: brain, mind, experience and school. Washington, DC: National Academy.

Buckner RL, Wheeler ME (2001) The cognitive neuroscience of remembering. Nat Rev Neurosci 2:624-634.

Burton H, Sinclair RJ (2000) Attending to and remembering tactile stimuli: a review of brain imaging data and single-neuron responses. J Clin Neurophysiol 17:575-591.

Diekelmann S, Born J (2010) The memory function of sleep. Nat Rev Neurosci 11:114-126.

Driver J, Noesselt T (2008) Multisensory interplay reveals crossmodal influences on 'sensory-specific' brain regions, neural responses, and judgments. Neuron 57:11-23.

Ernst MO (2007) Learning to integrate arbitrary signals from vision and touch. J Vis 7:7.1-714.

Fletcher PC, Henson RN (2001) Frontal lobes and human memory: insights from functional neuroimaging. Brain 124:849-881.

Frankland PW, Bontempi B (2006) Fast track to the medial prefrontal cortex. Proc Natl Acad Sci U S A 103:509-510.

Fredembach B, de Boisferon AH, Gentaz E (2009) Learning of arbitrary association between visual and auditory novel stimuli in adults: the "bond effect" of haptic exploration. PLoS One 4:e4844.

Gallace A, Spence C (2009) The cognitive and neural correlates of tactile memory. Psychol Bull 135:380-406.

Gilboa A, Alain C, He Y, Stuss DT, Moscovitch M (2009) Ventromedial prefrontal cortex lesions produce early functional alterations during remote memory retrieval. J Neurosci 29:4871-4881.

Harris JA, Petersen RS, Diamond ME (2001) The cortical distribution of sensory memories. Neuron 30:315-318.

Kan IP, Alexander MP, Verfaellie M (2009) Contribution of prior semantic knowledge to new episodic learning in amnesia. J Cogn Neurosci 21:938-944.

Kikyo H, Ohki K, Miyashita Y (2002) Neural correlates for feeling-of-knowing: an fMRI parametric analysis. Neuron 36:177-186.

Kim RS, Seitz AR, Shams L (2008) Benefits of stimulus congruency for multisensory facilitation of visual learning. PLoS One 3:e1532.

Kumaran D, Summerfield JJ, Hassabis D, Maguire EA (2009) Tracking the emergence of conceptual knowledge during human decision making. Neuron 63:889-901.

Lasry N, Aulls MW (2007) The effect of multiple internal representations on context-rich instruction. Am J Phys 75:1030-1037.

Maguire EA (2001) Neuroimaging studies of autobiographical event memory. Philos Trans R Soc Lond B Biol Sci 356:1441-1451.

Maguire EA, Frith CD, Morris RG (1999) The functional neuroanatomy of comprehension and memory: the importance of prior knowledge. Brain 122:1839-1850.

Mar RA (2004) The neuropsychology of narrative: story comprehension, story production and their interrelation. Neuropsychologia 42:1414-1434.

Marr D (1970) A theory for cerebral neocortex. Proc R Soc Lond B Biol Sci 176:161-234.

McClelland JL, McNaughton BL, O’Reilly RC (1995) Why there are complementary learning systems in the hippocampus and neocortex: insights from the successes and failures of connectionist models of learning and memory. Psychol Rev 102:419-457.

Miyashita Y (2004) Cognitive memory: cellular and network machineries and their top-down control. Science 306:435-440.

Moscovitch M, Nadel L, Winocur G, Gilboa A, Rosenbaum RS (2006) The cognitive neuroscience of remote episodic, semantic and spatial memory. Curr Opin Neurobiol 16:179-190.

Nyberg L, Habib R, McIntosh AR, Tulving E (2000) Reactivation of encoding-related brain activity during memory retrieval. Proc Natl Acad Sci U S A 97:11120-11124.

Patel VL, Yoskowitz NA, Arocha JF (2009) Towards effective evaluation and reform in medical education: a cognitive and learning sciences perspective. Adv Health Sci Educ Theory Pract 14:791-812.

Peters RM, Hackeman E, Goldreich D (2009) Diminutive digits discern delicate details: fingertip size and the sex difference in tactile spatial acuity. J Neurosci 29:15756-15761.

Peyrache A, Khamassi M, Benchenane K, Wiener SI, Battaglia FP (2009) Replay of rule-learning related neural patterns in the prefrontal cortex during sleep. Nat Neurosci 12:919-926.

Rasch B, Born J (2007) Maintaining memories by reactivation. Curr Opin Neurobiol 17:698-703.

Ridderinkhof KR, Ullsperger M, Crone EA, Nieuwenhuis S (2004) The role of the medial frontal cortex in cognitive control. Science 306:443-447.

Shams L, Seitz AR (2008) Benefits of multisensory learning. Trends Cogn Sci 12:411-417. 
Staresina BP, Gray JC, Davachi L (2009) Event congruency enhances episodic memory encoding through semantic elaboration and relational binding. Cereb Cortex 19:1198-1207.

Summerfield C, Koechlin E (2008) A neural representation of prior information during perceptual inference. Neuron 59:336-347.

Takashima A, Petersson KM, Rutters F, Tendolkar I, Jensen O, Zwarts MJ, McNaughton BL, Fernández G (2006) Declarative memory consolidation in humans: a prospective functional magnetic resonance imaging study. Proc Natl Acad Sci U S A 103:756-761.

Takashima A, Nieuwenhuis IL, Rijpkema M, Petersson KM, Jensen O, Fernández G (2007) Memory trace stabilization leads to large-scale changes in the retrieval network: a functional MRI study on associative memory. Learn Mem 14:472-479.

Takashima A, Nieuwenhuis IL, Jensen O, Talamini LM, Rijpkema M, Fernández G (2009) Shift from hippocampal to neocortical centered retrieval network with consolidation. J Neurosci 29:10087-10093.

Takehara-Nishiuchi K, McNaughton BL (2008) Spontaneous changes of neocortical code for associative memory during consolidation. Science 322:960-963.

Takehara-Nishiuchi K, Nakao K, Kawahara S, Matsuki N, Kirino Y (2006) Systems consolidation requires postlearning activation of NMDA receptors in the medial prefrontal cortex in trace eyeblink conditioning. J Neurosci 26:5049-5058.

Tse D, Langston RF, Kakeyama M, Bethus I, Spooner PA, Wood ER, Witter MP, Morris RG (2007) Schemas and memory consolidation. Science 316:76-82.
Tzourio-Mazoyer N, Landeau B, Papathanassiou D, Crivello F, Etard O, Delcroix N, Mazoyer B, Joliot M (2002) Automated anatomical labeling of activations in SPM using a macroscopic anatomical parcellation of the MNI MRI single-subject brain. Neuroimage 15:273-289.

van Kesteren MT, Fernández G, Norris DG, Hermans EJ (2010) Persistent schema-dependent hippocampal-neocortical connectivity during memory encoding and postencoding rest in humans. Proc Natl Acad Sci U S A 107:7550-7555.

von Kriegstein K, Giraud AL (2006) Implicit multisensory associations influence voice recognition. PLoS Biol 4:e326.

Wang SH, Morris RG (2010) Hippocampal-neocortical interactions in memory formation, consolidation, and reconsolidation. Annu Rev Psychol 61:49-79, C1-C4.

Weibull A, Björkman A, Hall H, Rosén B, Lundborg G, Svensson J (2008) Optimizing the mapping of finger areas in primary somatosensory cortex using functional MRI. Magn Reson Imaging 26:1342-1351.

Wheeler ME, Petersen SE, Buckner RL (2000) Memory's echo: vivid remembering reactivates sensory-specific cortex. Proc Natl Acad Sci U S A 97:11125-11129.

Yuval-Greenberg S, Deouell LY (2009) The dog's meow: asymmetrical interaction in cross-modal object recognition. Exp Brain Res 193:603-614.

Zwaan RA, Radvansky GA (1998) Situation models in language comprehension and memory. Psychol Bull 123:162-185. 